\title{
3-Hydroxy-3-methylglutaryl-coenzyme A reductase inhibitor simvastatin ameliorates renal fibrosis through HOXA13-USAG-1 pathway
}

\author{
Yoshifumi Hamasaki ${ }^{1,2}$, Kent Doi ${ }^{1}$, Koji Okamoto ${ }^{1}$, Hideaki ljichi ${ }^{3}$, George Seki ${ }^{1}$, Rui Maeda-Mamiya ${ }^{1}$, Toshiro Fujita ${ }^{1}$ \\ and Eisei Noiri ${ }^{1,4}$
}

Epidemiological data have suggested that 3-hydroxy-3-methylglutaryl-coenzyme A reductase inhibitors (statins) prevent the progression of chronic kidney diseases (CKDs), whereas the precise mechanism explaining in vitro to in vivo is missing. This study is aimed at exploring a new mechanism of action by statins on renal fibrosis, a hallmark of CKD, using mouse renal fibrosis model in vivo and Madin-Darby canine kidney (MDCK) cells expressing USAG-1 in vitro. C57/BL6 mice fed a $0.2 \%$ adenine-containing diet for 4 weeks developed renal dysfunction accompanied with severe tubulointerstitial fibrosis. Subsequent simvastatin (SIM) treatment $(50 \mathrm{mg} / \mathrm{kg}$ per day) for 2 weeks significantly suppressed fibrosis progression. We found that SIM enhanced bone morphogenetic protein-7 (BMP-7)-mediated anti-fibrotic signaling with the reduced expression of uterine sensitization-associated gene-1 (USAG-1), a BMP-7 antagonist produced by renal distal tubular epithelial cells. Therefore, MDCK cells were incubated with transforming growth factor- $\beta 1$ and showed increased expression of USAG-1 and $\alpha$-smooth muscle actin; SIM significantly reduced them. SIM significantly increased E-cadherin expression. Gene knockdown experiments using MDCK suggested that homeobox protein Hox-A13 (HOXA13) played a suppressive role in the USAG-1 gene and thus SIM reduced USAG-1 by increasing HOXA13 expression. The data from our study demonstrate that SIM, one of statins, contributes to prevent the progression of renal fibrosis by upregulating BMP-7-mediated anti-fibrotic signaling and that one aspect of crucial efficacies is achieved by regulating HOXA13 and USAG-1. HOXA13-USAG-1 pathway is a newly identified mechanism in renal fibrosis and will be a new therapeutic target for preventing renal fibrosis progression in CKDs.

Laboratory Investigation (2012) 92, 1161-1170; doi:10.1038/labinvest.2012.71; published online 23 April 2012

KEYWORDS: BMP-7; HOXA13; statin; TGF- $\beta 1$; USAG-1

Chronic kidney disease (CKD) has recently been recognized as a public health problem. Its incidence and prevalence continue to increase, entailing poor outcomes and high costs. $^{1,2}$ Although CKD progresses slowly, it eventually evolves to end-stage renal disease, which necessitates renal replacement therapy such as dialysis or kidney transplantation. Moreover, CKD is well known to contribute strongly to cardiovascular disease and high mortality. ${ }^{3}$ Unfortunately, no treatment can prevent the progression of CKD completely.

The best predictive factor for progression of CKD to endstage renal disease is not the etiology of glomerular injury, but the degree of tubulointerstitial damage such as fibrosis and inflammatory cell infiltration. ${ }^{4-7}$ Tubulointerstitial fibrosis is a common pathological change occurring in association with chronic kidney damage. ${ }^{8}$ Fibrosis is induced and accelerated by myofibroblasts in the renal interstitium, thereby producing an extracellular matrix. Interstitial myofibroblasts are reportedly derived from several sources, including proliferation of 'primary' fibroblasts, stem cells and renal tubular epithelial cells. ${ }^{9,10}$ Recently, it was reported that tubular epithelial cell cycle arrest at G2/M after kidney injury initiates to renal fibrosis progression. ${ }^{11}$ Two multifactorial

\footnotetext{
'Department of Nephrology and Endocrinology, and Hemodialysis and Apheresis, University Hospital, University of Tokyo, Tokyo, Japan; ${ }^{2} 22$ nd Century Medical and Research Center, University Hospital, University of Tokyo, Tokyo, Japan; ${ }^{3}$ Department of Gastroenterology, University Hospital, University of Tokyo, Tokyo, Japan and ${ }^{4} J a p a n$ Science and Technology Agency/Japan International Cooperation Agency (JST/JICA), Science and Technology Research Partnership for Sustainable Development (SATREPS), Tokyo, Japan

Correspondence: E Noiri, MD, PhD, Department of Nephrology and Endocrinology, and Hemodialysis and Apheresis, University of Tokyo, 7-3-1 Hongo, Bunkyo, Tokyo 113-8655, Japan. 
growth factors--transforming growth factor- $\beta 1$ (TGF- $\beta 1$ ) and bone morphogenic protein-7 (BMP-7)-are the key molecules to determine the fate of kidney; the balance between TGF- $\beta 1$ and BMP-7 signaling determines whether renal fibrosis is accelerated or not.

Results of clinical and basic studies suggest that 3-hydroxy3-methylglutaryl-coenzyme A reductase inhibitors (statins) can preserve renal function and suppress renal fibrosis. ${ }^{12}$ Some benefits of statins are achieved by a cholesterollowering independent effect. Potential pleiotropic effects of statins for renal injury, such as inhibition of isoprenoid synthesis, antioxidant effects, improvement of endothelial function and anti-inflammation, are expected from previous reports. ${ }^{12-15}$ Isoprenoids are necessary to activate small guanosine triphosphate binding proteins such as Ras homolog gene family member A. In vitro studies have shown that these small guanosine triphosphate binding proteins mediate TGF- $\beta 1$ signaling and accelerate renal fibrosis; ${ }^{16}$ statins suppress renal fibrosis by inhibiting Ras homolog gene family member A-mediated pathway. ${ }^{16,17}$ Although several mechanisms of renal preservation by statins have been suggested, no report has described an investigation of whether statins contributes to preventing renal fibrosis by modulating BMP-7-dependent anti-fibrotic pathway.

Recently, it has been recognized that uterine sensitizationassociated gene-1 (USAG-1), a dominant antagonist of BMP-7, contributes to renal injury. ${ }^{18,19}$ In previous studies, it was demonstrated that USAG-1 is expressed in renal distal tubular epithelial cells, and USAG-1 knockout mice showed reduced tubulointerstitial fibrosis in a unilateral ureter obstruction model. ${ }^{18,19}$ Nevertheless, little is known of whether suppressing USAG-1 by therapeutic agents will reduce renal fibrosis.

In this study, we examined the protective effect of simvastatin (SIM), a representative of statins, on a mouse renal interstitial fibrosis model, which is induced by feeding mice with adenine-containing diet (Ad) for several weeks. ${ }^{20} \mathrm{We}$ also performed in vitro analysis with Madin-Darby canine kidney (MDCK) cells to identify the target of SIM and mechanism by which SIM upregulates BMP-7-mediated anti-fibrotic pathway.

\section{MATERIALS AND METHODS}

\section{Animal Experiments}

Eight-week-old female C57BL/6 mice were used in this experiment. The animals were kept in specific pathogenfree conditions at our animal facility. All experiments were conducted in accordance with the NIH Guide for the Care and Use of Laboratory Animals (US Department of Health and Human Services Public Health Services, National Institutes of Health, NIH Publication No. 86-23, 1985) and approved by the University of Tokyo Institutional Review Board. They were allowed food and water ad libitum. Both the SIM-treated group (Ad + SIM group) and -untreated group (Ad group) were fed a $0.2 \%(\mathrm{w} / \mathrm{w})$ adenine-containing diet for 4 or 6 weeks. From 2 weeks after the start of Ad, the Ad + SIM group was given SIM (generously provided by MSD KK, Tokyo, Japan) suspended in 5\% carboxyl methyl cellulose solution $50 \mathrm{mg} / \mathrm{kg}$ per day by oral gavage for 2 or 4 weeks; the Ad group was given 5\% carboxyl methyl cellulose solution only. On the basis of various different doses of SIM administration in previous studies on animal models of chronic renal disease, ${ }^{15,21-23}$ we decided the dose of SIM in this study, which is close to the median value of those of previous studies $(10-100 \mathrm{mg} / \mathrm{kg}$ per day). The normal diet group ( $\mathrm{N}$ group) was fed a normal diet and given water for 4 weeks. Blood samples were collected at every 2 weeks after the start of adenine-containing diet. The mice were killed at 4 weeks to collect specimens.

\section{Blood Chemistry}

Blood urea nitrogen (BUN) was measured using the ureaseindophenol method (Urea N B test; Wako Pure Chemical Industries, Osaka, Japan). Serum total cholesterol was measured using an enzymatic colorimetric method (Cholesterol E test; Wako). Alanine aminotransferase was measured using the pyruvate oxidase- $N$-ethyl- $N$-(2-hydroxy-3-sulfopropyl)$m$-toluidine (POP-TOOS) method with a Transaminase CII-test Wako (Wako Pure Chemical Industries).

Plasma creatinine measurements using high-performance liquid chromatography (HPLC) were conducted according to a previously described method with some modification. ${ }^{24}$ HPLC mobile phase $(200 \mathrm{mM}$ sodium acetate, $\mathrm{pH}$ 5.1) was freshly made and filtered $(0.2 \mu \mathrm{m})$ before use. The HPLC grade acidified acetonitrile (Kanto Chemical, Tokyo, Japan) was added to plasma $(5 \mu \mathrm{l})$, vortexed and centrifuged for $15 \mathrm{~min}$ at $13000 \mathrm{~g}, 4^{\circ} \mathrm{C}$. The supernatant fraction of each sample was transferred completely to a tube, evaporated to dryness and resuspended immediately in $125 \mu \mathrm{l}$ of HPLC mobile phase. Sample injections ( $50 \mu \mathrm{l}$ each) were performed with a $125 \times 4.6 \mathrm{~mm}^{2}$ cation exchange column (Shodex IC YS-50; Showa Denko KK, Tokyo, Japan). Isocratic HPLC was performed at a flow rate of $1 \mathrm{ml} / \mathrm{min}$. Thereafter, UV absorbance at $234 \mathrm{~nm}$ was monitored for $8 \mathrm{~min}$ after each injection. The creatinine peak was eluted at $6.0-6.8 \mathrm{~min}$, and integration was performed using software (D-2000Elite; Hitachi High-Technologies, Tokyo, Japan).

\section{Pathological Analysis}

Kidneys were collected at 4 weeks after starting adeninecontaining diet and fixed in $10 \%$ buffered formalin. Paraffin sections of $2 \mu \mathrm{m}$ were stained with Masson's trichrome. Immunohistochemistry for $\alpha$-smooth muscle actin ( $\alpha$-SMA) and $\mathrm{F} 4 / 80$ was performed as described previously. ${ }^{20}$ Immunohistochemistry for E-cadherin was performed using anti-E-cadherin antibody (Santa Cruz Biotechnology, Santa Cruz, CA, USA). The area of interstitial fibrosis and the area of $\alpha$-SMA-positive myofibroblast, E-cadherin-positive epithelial cell and F4/80-positive macrophage were evaluated using a computer-aided evaluation program (AIS; Imaging 
Research, St Catharines, Ontario, Canada) as described previously. ${ }^{25}$ Interstitial volume in the renal cortex was evaluated by a point counting method as described previously. ${ }^{26}$

\section{Cell Culture, RNA Interference and Overexpression of Homeobox Protein Hox-A13}

MDCK cells and human embryonic kidney cells (HEK293 cells) were obtained from the American Type Culture Collection, and cultured in Dulbecco's modied Eagle's medium (Gibco, Carlsbad, CA, USA) containing 10\% fetal bovine serum (FBS), $100 \mathrm{U} / \mathrm{ml}$ penicillin $\mathrm{G}$ and $100 \mu \mathrm{g} / \mathrm{ml}$ streptomycin, in a humidified atmosphere containing 5\% $\mathrm{CO}_{2}$ at $37^{\circ} \mathrm{C}$. The MDCK cells were seeded on 6- or 12-well culture plates and the HEK293 cells on $10 \mathrm{~cm}$ culture dishes in complete medium containing $10 \%$ FBS.

On the basis of the previous observation that homeobox protein Hox-A13 (HOXA13), a transcriptional factor, binds to USAG-1 coding DNA region and regulates USAG-1 expression in organogenesis, ${ }^{27}$ knockdown of HOXA13 gene in MDCK cells was conducted by RNA interference (RNAi). After reaching approximately $30-40 \%$ confluent, MDCK cells were transfected with either Stealth RNAi Negative Control Med GC (Invitrogen, Carlsbad, CA, USA) or with predesigned small interfering RNA targeted against canine HOXA13 (Invitrogen) using Lipofectamine 2000 Reagent (Invitrogen). The sense and antisense sequences of small interfering RNA targeted against HOXA13 are: $5^{\prime}$-UUUGU UUGUAGCAUAUUCCCGUUCG-3' ${ }^{\prime}$ and $5^{\prime}$-CGAACGGGAA UAUGCUACAAACAAA- $3^{\prime}$. Subsequently, the cells were kept in serum-free medium for $24 \mathrm{~h}$, and then they were placed into the $1 \%$ FBS-added medium containing recombinant human $5 \mathrm{ng} / \mathrm{ml}$ TGF- $\beta 1$ (Sigma-Aldrich, St Louis, MO, USA) with or without $0.2 \mu \mathrm{M}$ SIM for $72 \mathrm{~h}^{28,29}$ We decided the concentration of SIM to reduce toxicity and preserve efficacy of SIM based on the result of our preliminary experiments showing that MDCK cells were detached by $2 \mu \mathrm{M}$ SIM treatment and USAG-1 expression was not changed by $0.02 \mu \mathrm{M}$ SIM treatment (data not shown).

To obtain sufficient HOXA13 protein for electrophoretic mobility shift assay (EMSA) described below, HOXA13 expressing plasmid DNA was transfected to HEK293 cells. After reaching approximately $90 \%$ confluent, HEK293 cells were transfected with either human HOXA13 plasmid DNA (ORIGENE Technologies, Rockville, MD, USA) or control empty plasmid DNA (Agilent Technologies, La Jolla, CA, USA), using Lipofectamine 2000 Reagent (Invitrogen). Thereafter, they were placed into complete medium containing 10\% FBS for $72 \mathrm{~h}$.

\section{Quantitative PCR Analysis for USAG-1, BMP-7, HOXA13, TGF- $\beta 1$ and MCP-1 Expression}

Total RNA isolated from the harvested kidneys or MDCK cells were reverse-transcribed to cDNA using recombinant MLV reverse transcriptase and random hexamers (High Capacity cDNA Reverse Transcription Kit; Applied Biosystems,
Carlsbad, CA, USA). Transcripts encoding TGF- $\beta 1$ and monocyte chemoattractant protein-1 (MCP-1) were measured as described previously. ${ }^{20}$ Transcripts encoding BMP-7, USAG-1 and canine HOXA13 were measured using SYBR Green-based quantitative PCR with Power SYBR Green PCR Master Mix (Applied Biosystems) and a sequence detection system (Prism 7000; Applied Biosystems). Murine HOXA13 was measured using FastStart DNA Master SYBR Green 1 (Roche Applied Science, Indianapolis, IN, USA). The sequences of primers are as follows: murine USAG-1 (forward: $5^{\prime}$-GCAACAGCACCCTGAATCAAG-3'; reverse: 5'-TGTATTTGGTGGACCGCAGTT- ${ }^{\prime}$ ), murine BMP-7 (forward: $5^{\prime}$-TGTGGCAGAAAACAGCAGCA-3'; reverse: $5^{\prime}$-TCA GGTGCAATGATCCAGTCC- $3^{\prime}$ ), murine HOXA13 (forward: 5'-CCTCTGGAAGTCCACTCTGC-3'; reverse: 5'-TATAGGAGC TGGCGTCTGAA- $3^{\prime}$ ), canine USAG-1 (forward: $5^{\prime}$-GGAGGCA GGCATTTCAGTAA- $3^{\prime}$; reverse: $5^{\prime}$-TGTACTTGGTGGAACGCA GT-3'), canine BMP-7 (forward: $5^{\prime}$-CTGGTGTTCGACATCACA GC-3'; reverse: $5^{\prime}$-CATGAAGGGCTGCTTGTTCT-3'), canine HOXA13 (forward: $5^{\prime}$-GGGAGAAAGAAGCGCGTC-3'; reverse: $5^{\prime}$-CGTCGTGGCTGATATCCG-3' ${ }^{\prime}$ ) and canine glyceraldehyde3-phosphate dehydrogenase (GAPDH) (forward: $5^{\prime}$-TGAAGGT CGGTGTGAACGGATTTGGC-3'; reverse: 5'-CATGTAGGCCA TGAGGTCCACCAC- $3^{\prime}$ ). To normalize for variance in loaded cDNA, $18 \mathrm{~S}$ ribosomal RNA (18S rRNA) of mice and GAPDH of MDCK cells were amplified in a separate tube using TaqManand SYBR Green-based quantitative PCR, respectively. Probe primers for $18 \mathrm{~S}$ rRNA was obtained from Applied Biosystems. Standard curves were prepared for each gene and the 18S rRNA or GAPDH in each experiment to normalize the relative expression of the genes of interest to the $18 \mathrm{~S}$ rRNA or GAPDH control.

\section{Immunoblot Analysis for $\alpha$-SMA, E-Cadherin, Phosphorylated Smad 2/3, Phosphorylated Smad1/5/8 and HOXA13}

Protein samples were extracted from the whole kidney or MDCK cells with radio-immunoprecipitation assay buffer containing a protease inhibitor cocktail as described previously. ${ }^{25}$ After centrifugation of the samples, the supernatants were used as whole kidney lysates or whole cell lysates. The lysates were boiled in sample buffer containing $5 \%(\mathrm{w} / \mathrm{v})$ SDS with $20 \%(\mathrm{v} / \mathrm{v})$ 2-mercaptoethanol and separated on a $10-12 \%$ sodium dodecyl sulfate-polyacrylamide gel electrophoresis. After transferring proteins from the gel to a polyvinylidene difluoride membrane (GE Healthcare UK, Buckinghamshire, UK), immunoblot analysis was performed using 1:1000 diluted anti $\alpha$-SMA antibody (1A4; Sigma-Aldrich), 1:500 diluted anti E-cadherin antibody (Santa Cruz Biotechnology), 1:200 diluted anti-phosphorylated Smad2/3 (pSmad2/3) antibody (Santa Cruz Biotechnology), 1:200 diluted anti-phosphorylated Smad1/ 5/8 (pSmad1/5/8) antibody (Cell Signaling Technologies, Danvers, MA, USA). The chemiluminescent signal labeled using ECL Plus (GE Healthcare UK) was detected using a 
CCD camera system (LAS-4000min; Fuji Photo Film, Tokyo, Japan). The membrane was incubated at $50^{\circ} \mathrm{C}$ for $30 \mathrm{~min}$ in a stripping buffer to remove all the probes; it was re-probed with anti $\alpha$-tubulin antibody (Santa Cruz Biotechnology). Densitometric analysis of bands compared with the density of $\alpha$-tubulin was performed using the software Multi Gauge, ver. 3.1 (Fuji Photo Film).

\section{HOXA13 Binding Sites in USAG-1 Promoter}

HOXA13 DNA-binding domain has high affinity to the sequence containing AATAAA (core DNA sequence is TAA). ${ }^{27}$ We found such sequence within $2000 \mathrm{bp}$ upstream of canine USAG-1 coding region, according to the database search of NCBI (http://www.ncbi.nlm.nih.gov/). To investigate whether HOXA13 binds to this DNA sequence located in the upstream of USAG-1 coding region, we conducted EMSA.

A $40 \mathrm{bp}$ oligonucleotide sequence, which locates upstream of USAG-1 coding region (sequence position relative to the start codon of USAG-1 is -1932 to -1892) and contains the motif 'AATAAA' in the middle of its sequence, was synthesized and biotinylated ( 5 '-TTTCAGAAAGGATGTCAGAAT AAACATCTACATACAACCT-3'; Sigma-Aldrich). This oligonucleotide sequence was annealed with complementary oligonucleotide sequence (Sigma-Aldrich). Nuclear proteins were extracted from HEK293 cells transfected with either HOXA13 or control empty plasmid DNA using commercial kit (CelLytic NuCLEAR Extraction Kit; SigmaAldrich). EMSA was performed using the commercial kit (Panomics, Fremont, CA, USA) as described previously. ${ }^{30}$ Briefly, nuclear extracts were incubated with a biotin-labeled or -unlabeled $40 \mathrm{bp}$ oligonucleotide for $30 \mathrm{~min}$ at $15^{\circ} \mathrm{C}$, and HOXA13-bound oligonucleotide was separated from unbound oligonucleotide by electrophoresis on a $6 \% \mathrm{TBE}$ gel. After transfer to Biodyne B nylon membrane (Pall, Port Washington, NY, USA), the biotin-labeled bands were visualized using horseradish peroxidase-based chemiluminescence.

\section{Statistical Analysis}

Results are expressed as mean values \pm s.e.m. Differences among experimental groups were determined using one-way ANOVA with post hoc analysis. Differences for which $P<0.05$ were considered significant.

\section{RESULTS}

Effects of SIM on Renal Function and Histopathological Changes in Adenine-Induced Mouse Tubulointerstitial Injury Model

BUN levels of the adenine diet group were significantly higher than the normal diet group ( $\mathrm{N}$ group) 2 weeks after starting adenine diet ( $\mathrm{N}$ group, $17.9 \pm 1.0 \mathrm{mg} / \mathrm{dl} \quad(n=6)$; adenine diet group, $49.1 \pm 4.1 \mathrm{mg} / \mathrm{dl}(n=16))$. Mice of adenine diet group were equally divided into two groups, the vehicle-only group (Ad group) and the SIM treatment group (Ad + SIM group) at 2 weeks. The levels of BUN and plasma
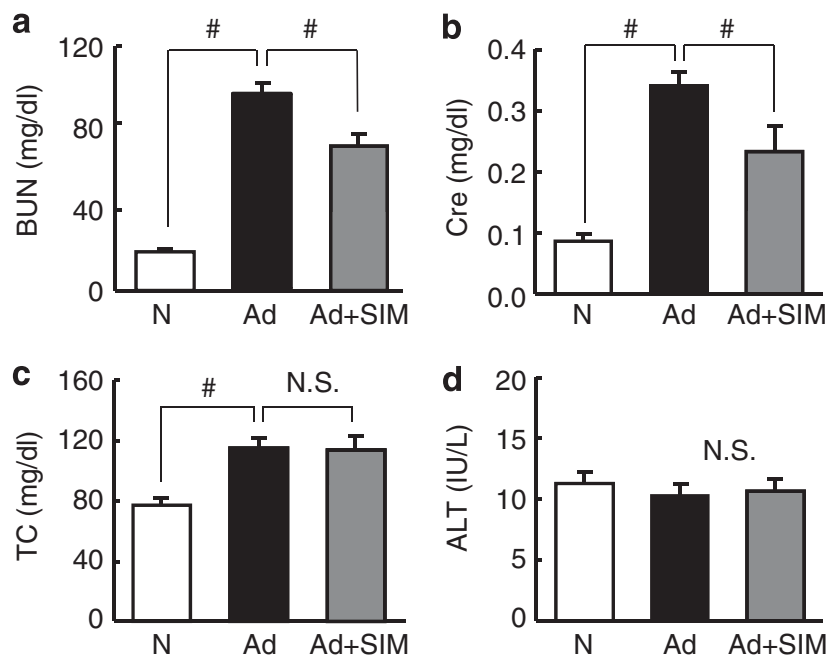

Figure 1 Effect of simvastatin (SIM) on mouse adenine-induced tubulointerstitial injury model. Blood urea nitrogen (BUN), plasma creatinine (Cre), serum total cholesterol (TC) and serum alanine aminotransferase (ALT) levels were measured at 4 weeks $(\mathbf{a}-\mathbf{d})$. $\mathrm{N}$ : normal diet; Ad: adenine diet; Ad + SIM: adenine diet and SIM treatment. Data are means \pm s.e.m.; $n=6-8$ in each group. ${ }^{\#} P<0.05$.

creatinine in the Ad + SIM group $(n=8)$ were significantly lower than the Ad group $(n=8)$ at 4 weeks (Figures 1a and $b$ ). When we examined the level of BUN in another set of animals, BUN levels in the Ad + SIM group were also significantly lower than the Ad group at 6 weeks (Ad group, $132.1 \pm 6.9 \mathrm{mg} / \mathrm{dl}(n=5) ; \mathrm{Ad}+$ SIM group, $115.5 \pm 5.4 \mathrm{mg} / \mathrm{dl}$ $(n=5))$. Because renal protective effect of SIM was observed as early as 4 weeks, we decided to evaluate the mechanism of action of SIM after 2-week treatment. Adenine administration increased serum total cholesterol levels measured at 4 weeks; however, SIM did not influence them (Figure 1c). These data show that SIM improved renal function independently from its cholesterol-lowering effect. Neither adenine administration nor SIM changed the serum alanine aminotransferase levels of the animals (Figure 1d). Pathological evaluation showed remarkable interstitial fibrosis, irregular renal tubular dilatation, increased $\alpha$-SMA expression and decreased E-cadherin expression in the kidney at 4 weeks (Figure 2a). Fibrotic area in Masson's trichrome stain was increased by adenine administration and improved by SIM treatment (Figure 2b). Interstitial volume evaluated by point counting method was also significantly increased in the Ad group compared with the $\mathrm{N}$ group and improved by SIM treatment (Figure 2c). Quantitative analyses on $\alpha$ SMA-positive area and E-cadherin-positive area revealed that SIM improved these histopathological changes (Figures $2 \mathrm{~d}$ and e). F4/80-positive inflammatory cell (macrophage) infiltration in the interstitium was increased by adenine administration and reduced by SIM (Figures $2 \mathrm{f}$ and g). The mRNA expression of MCP-1 in the kidney was increased by adenine administration; SIM treatment decreased it significantly (Figure $2 \mathrm{~h}$ ). 
a

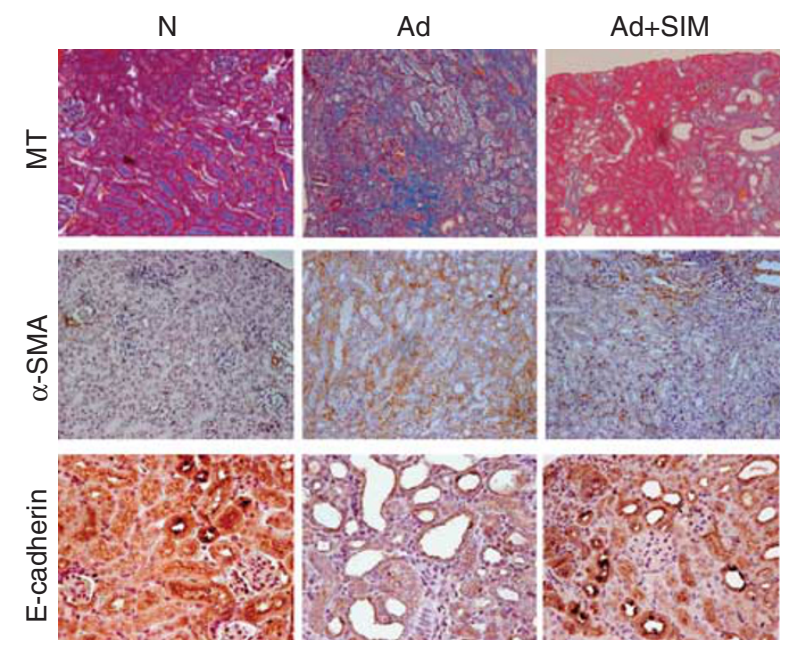

f

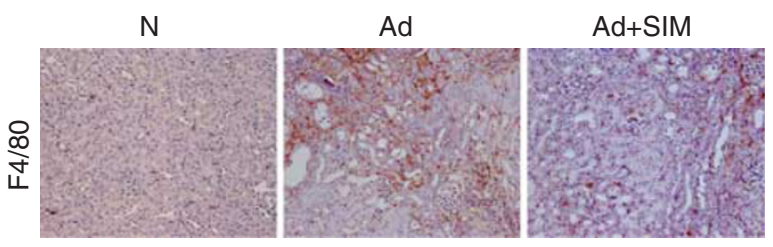

b
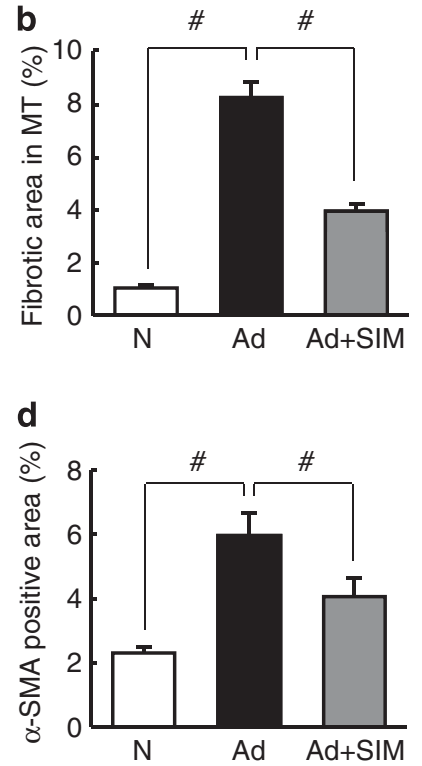

g

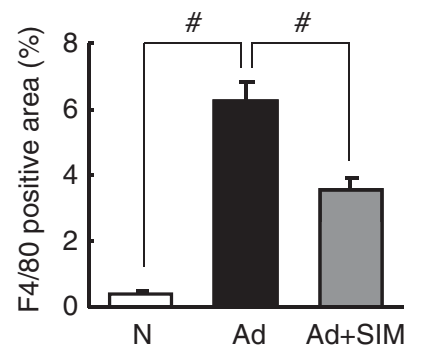

C

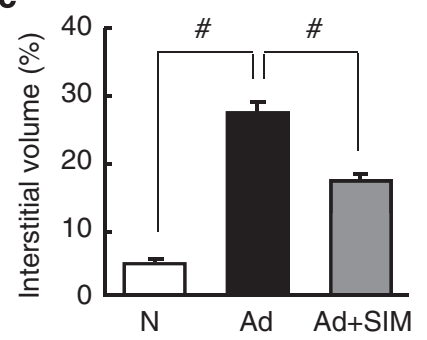

e

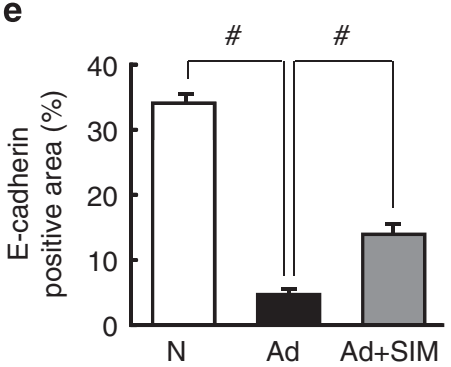

$\mathbf{h}$

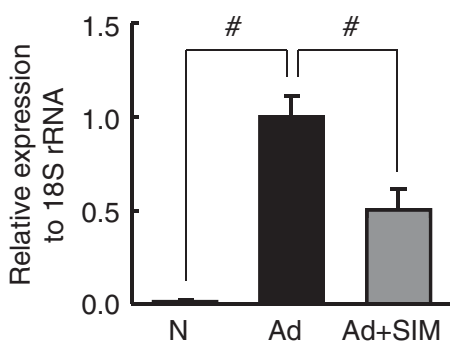

Figure 2 Renal pathological findings in mouse adenine-induced tubulointerstitial injury model and mRNA expression of monocyte chemoattractant protein-1 (MCP-1) in the kidney. Renal histological changes evaluated using Masson's trichrome (MT), immunohistochemistry of $\alpha$-smooth muscle actin $(\alpha$-SMA) and E-cadherin are shown (a). Original magnifications: $\times 100$ in MT; $\times 200$ in $\alpha$-SMA and E-cadherin. Fibrotic area in Masson's trichrome stain (b), interstitial volume in renal cortex evaluated by point counting method (c), positive staining area of $\alpha$-SMA (d) and E-cadherin (e) were quantified. Immunohistochemistry of F4/80-positive inflammatory cells (macrophages) is shown (f). Positive staining area of F4/80 was quantified (g). MCP-1 was evaluated using real-time polymerase chain reaction (PCR) (h). N: normal diet; Ad: adenine diet; $\mathrm{Ad}+\mathrm{SIM}$ : adenine diet and simvastatin treatment. Data are means \pm s.e.m.; $n=6-8$ in each group. ${ }^{\#} P<0.05$.

\section{Evaluation of the Molecules Associated with Fibrosis and Anti-Fibrosis in the Kidney}

The mRNA expression levels of TGF- $\beta 1$ in the kidney were increased by adenine administration and SIM treatment decreased them significantly (Figure 3a). Immunoblot analysis demonstrated that SIM significantly reduced the expression of phosphorylated Smad2/3 (Figure 3b) and $\alpha$-SMA (Figure 3c), which acts as a transducer of TGF- $\beta 1$-mediated signaling pathway and a marker of renal fibrosis progression.

BMP-7 gene expression levels were not changed by adenine administration or SIM treatment (Figure 3d). With regard to the report demonstrating that phosphorylation of Smad1/5/8 is associated with BMP-7 signaling in the kidney of mice, ${ }^{19}$ we found that phosphorylation levels of Smad1/5/8 were reduced in the Ad group and SIM significantly increased it (Figure 3e). SIM significantly increased the expression of E-cadherin, a phenotype marker of epithelial cells (Figure 3f). We further examined the expression of a BMP-7 antagonist,
USAG-1, and found that its mRNA expression was increased in the Ad group; SIM significantly reduced it (Figure 3g). HOXA13 is a transcriptional factor that negatively regulates USAG-1 expression. ${ }^{27}$ We observed that renal expression of HOXA13 was significantly increased in the Ad + SIM group compared with the Ad group (Figure $3 \mathrm{~h}$ ). These data indicate that SIM not only suppressed fibrotic pathway, but also enhanced anti-fibrotic pathway by downregulating a dominant BMP-7 antagonist USAG-1, and not by enhancing BMP-7 production. The effect of SIM for USAG-1 is, at least partly, mediated by its effect for HOXA13 upregulation.

\section{Effects of SIM on In Vitro Model of Fibrosis Using Distal Tubular Epithelial Cells}

We conducted in vitro experiments using MDCK cells to evaluate molecules expressed in renal distal tubular cells. $^{28,31,32}$ An in vitro model of fibrosis was induced by incubating MDCK cells with TGF- $\beta 1 .^{28,29}$ On quantitative 
a

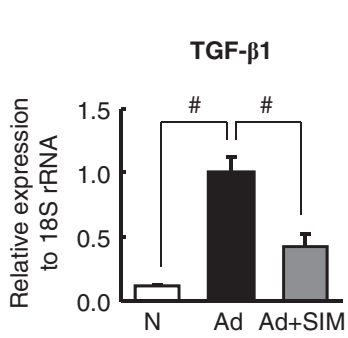

d

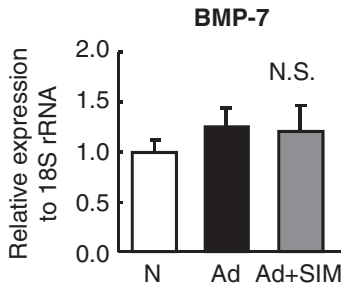

b

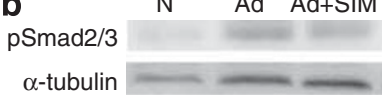

$$
3.0
$$

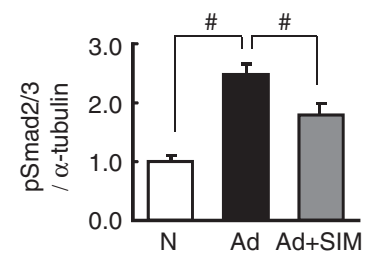

e
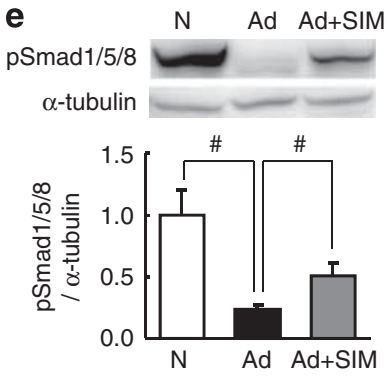

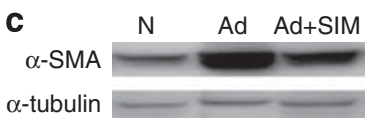

$\alpha$-tubulin
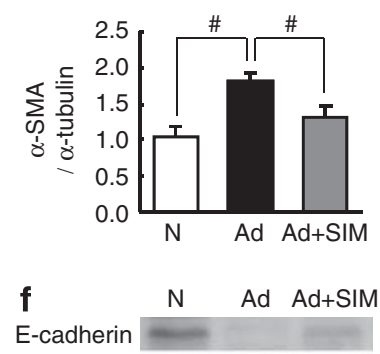

$\alpha$-tubulin

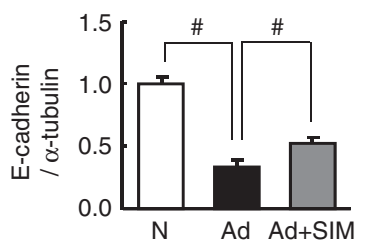

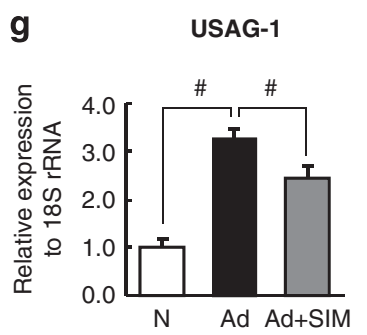

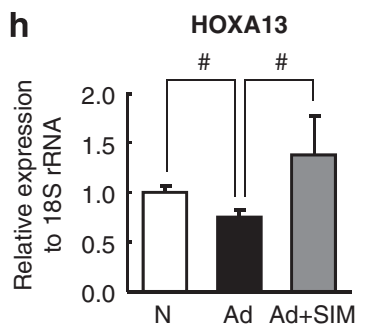

Figure 3 Expression of molecules associated with fibrosis and anti-fibrosis in mouse adenine-induced tubulointerstitial injury model. Expressions of molecules associated with fibrosis progression were evaluated by quantitative real-time polymerase chain reaction (PCR) (transforming growth factor- $\beta 1$ (TGF- $\beta 1$ ) (a)) and immunoblotting (phosphorylated Smad2/3 (pSmad2/3) (b) and $\alpha$-smooth muscle actin ( $\alpha$-SMA) (c)). Expressions of molecules associated with anti-fibrosis were evaluated by quantitative real-time PCR (bone morphogenetic protein-7 (BMP-7) (d)) and immunoblotting (phosphorylated Smad1/5/8 (pSmad1/5/8) (e) and E-cadherin (f)). Quantitative real-time PCR analysis examined USAG-1 expression (g). A transcriptional factor homeobox protein Hox-A13 (HOXA13), which negatively regulates USAG-1 expression, was examined by real-time PCR (h). N: normal diet; Ad: adenine diet; $\mathrm{Ad}+\mathrm{SIM}$ : adenine diet and simvastatin treatment. Data are means \pm s.e.m.; $n=6-8$ in each group. ${ }^{\#} P<0.05$. N: normal diet; Ad: adenine diet; $\mathrm{Ad}+\mathrm{SIM}$ : adenine diet and simvastatin treatment. Data are means \pm s.e.m.; $n=6-8$ in each group. ${ }^{\#} P<0.05$.

real-time PCR, the BMP-7 expression was not changed by either TGF- $\beta 1$ stimulation or SIM treatment (Figure $4 \mathrm{a}$ ). USAG- 1 expression in MDCK cells was increased by TGF- $\beta 1$; SIM decreased it (Figure $4 \mathrm{~b}$ ). We found that HOXA13 expression in MDCK cells was decreased by TGF- $\beta 1$, whereas SIM increased HOXA13 expression significantly (Figure 4c). On immunoblotting, $\alpha$-SMA expression was increased and E-cadherin was decreased significantly when MDCK cells were incubated with TGF- $\beta 1$, and SIM treatment improved them (Figures $4 \mathrm{~d}$ and e). We further conducted knockdown of HOXA13 by RNAi. The BMP-7 expression was not changed considerably as a result of RNAi for HOXA13 (Figure 4a, hatched bar). However, results show that the effect of SIM on USAG-1 expression was remarkably reversed by knockdown of HOXA13 gene (Figure 4b, hatched bar). The effect of SIM for $\alpha$-SMA and E-cadherin expression in MDCK cells was also canceled by knockdown of HOXA13 gene in significant level (Figures $4 \mathrm{~d}$ and e, hatched bar). These results suggest that SIM enhanced BMP-7-dependent anti-fibrotic signaling via HOXA13-USAG-1 pathway.

\section{Binding of HOXA13 to the Promoter Region of USAG-1 Gene}

In accordance with the previous report, ${ }^{33}$ transfection efficacy of plasmid DNA into MDCK cells was not so high in our experiment that HOXA13 protein could not be obtained sufficiently for EMSA. Therefore, we used HEK293 cells, which showed better transfection efficacy than MDCK cells. Overexpression of HOXA13 in HEK293 cells was confirmed by immunoblot analysis with the nuclear extract (Figure 5a). EMSA was conducted to examine whether HOXA13 protein in the nuclear extract bound to DNA sequence in the promoter region of USAG-1. When nuclear extract was incubated with both biotinylated and unlabeled competitor DNA probe, a shift of the intensive band was observed, 
a

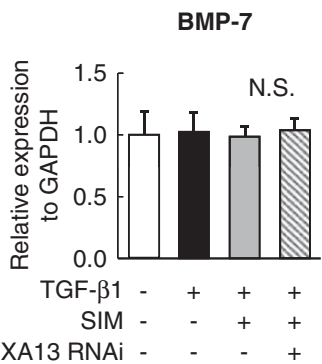

b

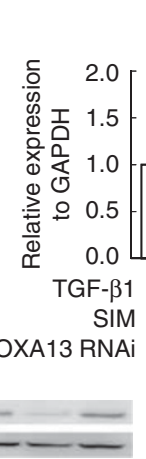

$$
\alpha \text {-tubulin }
$$

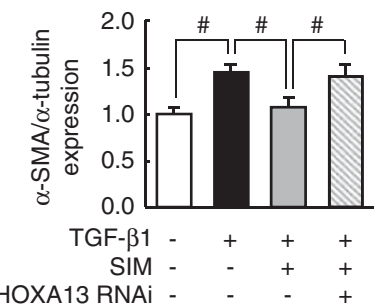

OXA13 RNAi -
USAG-1

\#\# \#

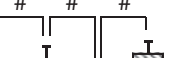

C

HOXA13
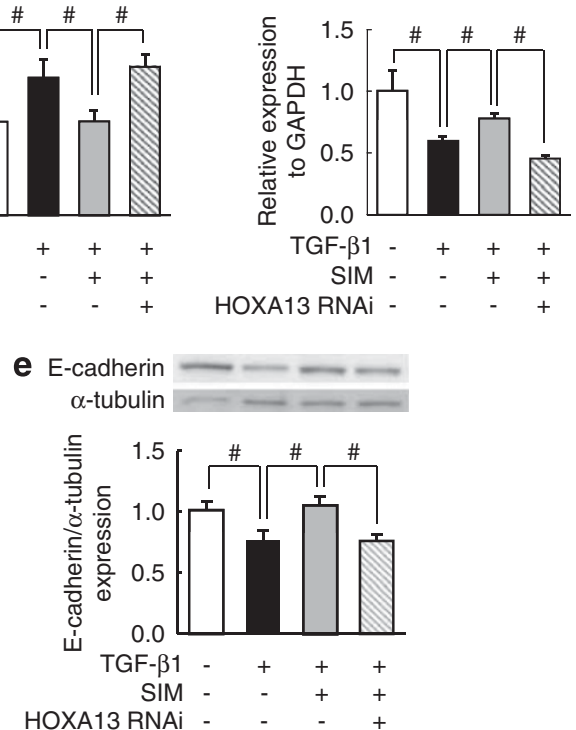

Figure 4 Expression of molecules associated with fibrosis and anti-fibrosis and homeobox protein Hox-A13 (HOXA13) in Madin-Darby canine kidney (MDCK) cells. Bone morphogenetic protein-7 (BMP-7) (a), USAG-1 (b) and HOXA13 (c) in vitro experiment with MDCK cells were evaluated using real-time polymerase chain reaction (PCR). Immunoblotting of $\alpha$-smooth muscle actin ( $\alpha$-SMA) (d) and E-cadherin (e) in vitro experiment with MDCK cells is shown. Knockdown of HOXA13 canceled the effect of simvastatin (SIM) for the expression of USAG-1 (b; hatched bar), $\alpha$-SMA (d; hatched bar) and E-cadherin (e; hatched bar). SIM: simvastatin treatment; HOXA13 RNAi: RNA interference for HOXA13. Data are means \pm s.e.m.; $n=9-12$ in each group, obtained from more than three independently conducted experiments. ${ }^{\#} P<0.05$.

a
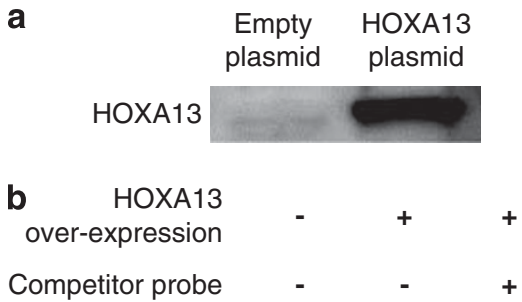

Figure 5 Electrophoretic mobility shift assay for homeobox protein Hox-A13 (HOXA13) binding site in the promoter region of USAG-1 gene. Nuclear HOXA13 protein expression in HOXA13-overexpressing HEK293 cells and control cells (empty plasmid) is shown (a). Electrophoretic mobility shift assay showed that HOXA13 protein bound to DNA probe that is identical to the sequence in the USAG-1 promoter region, and the competitor (unlabeled) DNA probe reduced the intensity of the positive band (b). indicating that HOXA13 binds to the promoter of USAG-1 gene (Figure $5 b$ ).

\section{DISCUSSION}

In this study, we found that SIM ameliorated renal fibrosis through HOXA13-USAG-1 pathway for the first time. In several large clinical trials, statins preserved or improved renal function. ${ }^{34-39}$ Although results of basic studies suggest that statins have pleiotropic effects in a cholesterol-lowering effect-independent manner, ${ }^{12-17}$ the mechanisms have not been sufficiently revealed yet. Furthermore, no report in the relevant literature has described examination of whether statins modulate BMP-7- and USAG-1-mediated pathway, which counteracts the TGF- $\beta 1$-mediated fibrotic pathway. This study demonstrates that SIM contributes to suppression of renal fibrosis by modulating the BMP-7-dependent antifibrotic pathway in vivo independently from its cholesterollowering effect.

Adenine-induced renal fibrosis model using rats was originally reported several decades ago, ${ }^{40}$ and we recently modified this model with C57BL/6 mice. ${ }^{20}$ Adenine is oxidized to 2,8-dihydroxyadenine by xanthine dehydrogenase and 2,8dihydroxyadenine will be precipitated in renal tubules because of its low solubility. Deposition of 2,8-dihydroxyadenine in renal tubules degenerates renal tubular epithelial cells and causes inflammatory injury with subsequent fibrotic 
changes. Renal fibrosis in this model was evaluated in two different measurements of interstitial volume and fibrotic area stained as blue by Masson's trichrome (Figure 2b and c). Not only interstitial volume, which is reported to be approximately $4-9 \%$ in the normal renal cortex,${ }^{26,41}$ but also fibrotic area was significantly increased by adenine-containing diet in accordance with previous reports including unilateral ureteral obstruction model..$^{20,26}$

Results from our in vivo and in vitro experiments indicate that SIM enhanced anti-fibrotic pathway by suppressing USAG-1, not by upregulating BMP-7. Actually, TGF- $\beta 1$ has been identified as the dominant inducer of renal fibrosis, ${ }^{10,42}$ whereas BMP-7 signaling counteracts TGF- $\beta 1$-mediated fibrotic pathway within tubular epithelial cells. ${ }^{43,44}$ In the adult kidney, BMP-7 is produced in distal tubular epithelial cells and secreted from these cells to contribute to repair of chronic tubular injury. ${ }^{45-47}$ Among the several BMP-7 antagonists detected in the kidney, ${ }^{48}$ USAG-1 acts as a dominant BMP-7 antagonist and contributes strongly to renal tubular injury. ${ }^{19}$ Direct effects of USAG-1 on renal disease have been reported by using systemic USAG-1 knockout mice. Yanagita et al ${ }^{19}$ showed that USAG-1 knockout mice exhibited prolonged survival and preserved renal function in acute and chronic renal injury models. Tanaka et $a l^{49}$ demonstrated that ablation of USAG-1 gene in Alport syndrome model mice led to substantial attenuation of disease progression. Because USAG-1 is strongly expressed during development besides in the adult kidney, renal tubule-specific USAG-1 knockdown by using recently advanced technique such as Tet-On/Off system will provide more direct evidence of the role of USAG-1.

In this study, we observed decreased expression of TGF- $\beta 1$ and $\mathrm{pSmad} 2 / 3$ by SIM treatment in vivo (Figures $3 \mathrm{a}$ and $\mathrm{b}$ ). The marker of infiltrative macrophage and MCP-1 expression were attenuated by SIM (Figure $2 \mathrm{~h}$ ). Therefore, SIM appears to show anti-fibrotic effect in the kidney by acting on multiple pathways; USAG-1 affected BMP-7-mediated antifibrotic pathway and other mechanisms of renal fibrosis including TGF- $\beta 1$-mediated fibrotic pathway. The balance between TGF- $\beta 1$-mediated signaling (so-called epithelialto-mesenchymal transition (EMT)) and BMP-7-mediated signaling (so-called mesenchymal-to-epithelial transition (MET) ) will determine the fate of cell to $\alpha$-SMA-positive fibroblast or E-cadherin-positive epithelial cell, respectively. ${ }^{50}$ In this study, molecules related to renal fibrosis progression, TGF- $\beta 1, \mathrm{pSmad} 2 / 3$ and $\alpha$-SMA, were all increased in fibrotic kidney and reduced by SIM treatment. On the other hand, pSmad1/5/8 and E-cadherin expression were decreased in fibrotic kidney and increased by SIM, although BMP-7 levels did not change. USAG-1, a dominant antagonist of BMP-7, was increased in fibrotic kidney, and reduced by SIM. These data indicate that SIM put the EMT/MET balance towards MET in the fibrotic kidney, at least partly, by decreasing USAG-1 without any direct effect on BMP-7 (Figure 6). In vitro experiments using MDCK cells partially support

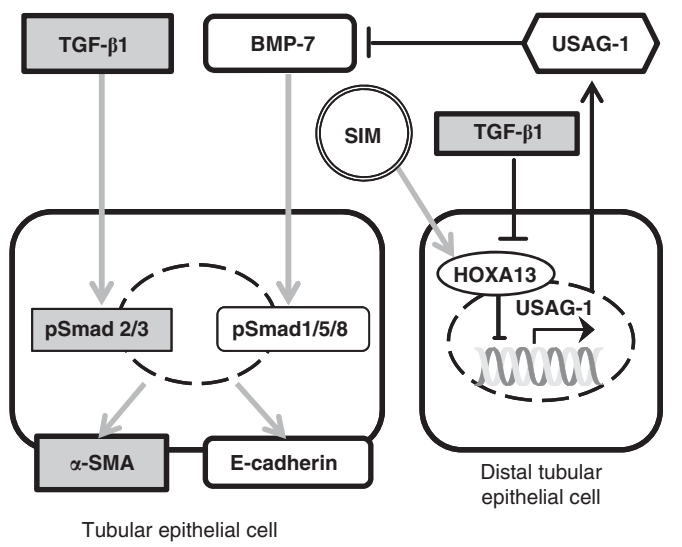

Figure 6 Schematic interpretation of fibrotic and anti-fibrotic pathway from homeobox protein Hox-A13 (HOXA13)-USAG-1 axis. Transforming growth factor- $\beta 1$ (TGF- $\beta 1$ ) and bone morphogenetic protein-7 (BMP-7) cause phosphorylation of $\mathrm{Smad} 2 / 3$ and Smad1/5/8, respectively. These intracellular proteins move into the nucleus and activate downstream gene transcriptions. The balance between TGF- $\beta 1$-mediated fibrotic signaling and BMP-7-mediated anti-fibrotic signaling will determine the fate of cell to myofibroblast ( $\alpha$-smooth muscle actin ( $\alpha$-SMA)-positive) or epithelial cell (E-cadherin-positive). USAG-1 secreted from renal distal tubular epithelial cells acts as a dominant antagonist of BMP-7 and HOXA13 contributes to upregulate BMP-7-mediated anti-fibrotic signaling by suppressing USAG-1 expression. Simvastatin (SIM) promotes BMP-7-mediated anti-fibrotic signaling and ameliorates renal fibrosis by upregulating HOXA13 expression.

in vivo findings described above (Figure 4). Multiple intracellular signaling including not only Smad phosphorylations, but also downstream intranuclear molecules of Smurf, SnoN, STAT3 and $\beta$-catenin may indicate TGF- $\beta 1$ - and BMP-7mediated pathway activations. Further investigation for these intracellular/intranuclear molecules in vitro that will give us more information about the protective effect of SIM is necessary.

Although it has been shown that systemic administration of BMP-7 is effective to reduce renal fibrosis in experiments with an animal renal fibrosis model, ${ }^{43,47,51}$ it remains to be resolved whether systemic adverse effects such as ectopic bone formation might occur after systemic administration of BMP-7. However, anti-fibrosis treatment targeting USAG-1 is reasonable to prevent systemic adverse effects because USAG-1 is expressed restrictedly in renal distal tubular epithelial cells in adult tissues. Drug discovery targeting USAG-1 might provide a new therapeutic agent against CKD, which suppresses the progression of renal dysfunction and has less systemic adverse effect.

Previous report using in situ hybridization showed that USAG-1 mRNA expression is limited in distal tubular cells of adult kidney. ${ }^{18,19,49}$ Therefore, we conducted in vitro experiments using MDCK cells (derived from distal tubular epithelial cells) to analyze the expression of USAG-1. We further demonstrated that SIM suppresses USAG-1 expression by the action of modulating HOXA13, a transcriptional factor encoded by a homeobox gene: HOXA13. Mammalian HOX genes, including HOXA13, serve an important role 
during embryogenesis. It has been demonstrated that HOXA13 is associated with limb formation and genitourinary development. Mutations in the HOXA13 gene cause abnormalities in the development of limb and genitourinary structures. ${ }^{52,53}$ Targeted disruption of the HOXA13 gene leads to a specific forelimb and hindlimb autopodal phenotype. ${ }^{54}$ Recently, Knosp et al ${ }^{27}$ identified multiple HOXA13 binding sites within the promoter region of USAG-1 gene. They further demonstrated dosage-dependent suppression of USAG-1 expression by HOXA13 using luciferase assay and elevated USAG-1 expression in HOXA13 homozygous mutant limbs. In our in vitro experiments with MDCK cells, the effect of SIM on USAG-1 was cancelled by knockdown of HOXA13 gene, indicating that HOXA13 negatively modulates USAG-1 expression. We further demonstrated that HOXA13 binds to the USAG-1 promoter region by EMSA (Figure 5).

Reportedly, HOXA13 also inhibits TGF- $\beta 1 /$ Smadmediated transcriptional activity in addition to the suppressive effect on USAG-1. This indicates that HOXA13 contributes to not only enhance BMP-7-mediated antifibrotic pathway, but suppress TGF- $\beta 1$-mediated fibrotic pathway. ${ }^{55}$ Further investigation is necessary to clarify the role of HOXA13 in the pathogenesis of renal fibrosis. Because HOXA13 plays a role in embryogenesis and is expressed in many organs, kidney-specific knockout of HOXA13 may give us more direct evidence for involvement of HOXA13 in USAG-1 regulation and renal fibrosis.

In conclusion, SIM contributes to preventing the progression of renal fibrosis by accelerating BMP-7-mediated anti-fibrotic effect in a mouse tubulointerstitial injury model. In vitro analysis with MDCK cells revealed that the protective effect of SIM is achieved by suppressing USAG-1 expression via a transcriptional factor HOXA13. This study demonstrates not only that the protective effects of statin for progressive renal fibrosis are associated with HOXA13 and USAG-1, but that HOXA13-USAG-1 pathway would be a novel drug target against the progression of renal fibrosis.

\section{ACKNOWLEDGEMENT}

We thank Ms. Haba for her skilled assistance.

\section{DISCLOSURE/CONFLICT OF INTEREST}

The authors declare no conflict of interest.

1. Coresh J, Selvin E, Stevens LA, et al. Prevalence of chronic kidney disease in the United States. JAMA 2007;298:2038-2047.

2. Trivedi $\mathrm{H}$. Cost implications of caring for chronic kidney disease: are interventions cost-effective? Adv Chronic Kidney Dis 2010;17: 265-270.

3. Go AS, Chertow GM, Fan D, et al. Chronic kidney disease and the risks of death, cardiovascular events, and hospitalization. N Engl J Med 2004;351:1296-1305.

4. Remuzzi G, Benigni A, Remuzzi A. Mechanisms of progression and regression of renal lesions of chronic nephropathies and diabetes. J Clin Invest 2006;116:288-296.

5. Risdon RA, Sloper JC, De Wardener HE. Relationship between renal function and histological changes found in renal-biopsy specimens from patients with persistent glomerular nephritis. Lancet 1968;2: 363-366.

6. Mackensen-Haen S, Bader R, Grund KE, et al. Correlations between renal cortical interstitial fibrosis, atrophy of the proximal tubules and impairment of the glomerular filtration rate. Clin Nephrol 1981;15:167-171.

7. Nath KA. Tubulointerstitial changes as a major determinant in the progression of renal damage. Am J Kidney Dis 1992;20:1-17.

8. Remuzzi G, Bertani T. Pathophysiology of progressive nephropathies. N Engl J Med 1998;339:1448-1456.

9. Kalluri R, Neilson EG. Epithelial-mesenchymal transition and its implications for fibrosis. J Clin Invest 2003;112:1776-1784.

10. Liu Y. Epithelial to mesenchymal transition in renal fibrogenesis: pathologic significance, molecular mechanism, and therapeutic intervention. J Am Soc Nephrol 2004;15:1-12.

11. Yang L, Besschetnova TY, Brooks CR, et al. Epithelial cell cycle arrest in G2/M mediates kidney fibrosis after injury. Nat Med 2010;16:535-543; $531 p$ following 143 .

12. Kassimatis TI, Konstantinopoulos PA. The role of statins in chronic kidney disease (CKD): friend or foe? Pharmacol Ther 2009;122:312-323.

13. Fried LF. Effects of HMG-CoA reductase inhibitors (statins) on progression of kidney disease. Kidney Int 2008;74:571-576.

14. Liao JK. Isoprenoids as mediators of the biological effects of statins. J Clin Invest 2002;110:285-288.

15. Vieira Jr JM, Mantovani E, Rodrigues LT, et al. Simvastatin attenuates renal inflammation, tubular transdifferentiation and interstitial fibrosis in rats with unilateral ureteral obstruction. Nephrol Dial Transplant 2005;20:1582-1591.

16. Patel S, Mason RM, Suzuki J, et al. Inhibitory effect of statins on renal epithelial-to-mesenchymal transition. Am J Nephrol 2006;26:381-387.

17. Rodrigues-Diez R, Carvajal-Gonzalez G, Sanchez-Lopez E, et al. Pharmacological modulation of epithelial mesenchymal transition caused by angiotensin II. Role of ROCK and MAPK pathways. Pharm Res 2008;25:2447-2461.

18. Yanagita M, Oka M, Watabe $T$, et al. USAG-1: a bone morphogenetic protein antagonist abundantly expressed in the kidney. Biochem Biophys Res Commun 2004;316:490-500.

19. Yanagita $M$, Okuda $T$, Endo $S$, et al. Uterine sensitization-associated gene-1 (USAG-1), a novel BMP antagonist expressed in the kidney, accelerates tubular injury. J Clin Invest 2006;116:70-79.

20. Tanaka T, Doi K, Maeda-Mamiya R, et al. Urinary L-type fatty acidbinding protein can reflect renal tubulointerstitial injury. Am J Pathol 2009;174:1203-1211.

21. Chade AR, Zhu XY, Grande JP, et al. Simvastatin abates development of renal fibrosis in experimental renovascular disease. J Hypertens 2008;26:1651-1660.

22. Ivanovski O, Szumilak D, Nguyen-Khoa T, et al. Effect of simvastatin in apolipoprotein $\mathrm{E}$ deficient mice with surgically induced chronic renal failure. J Urol 2008;179:1631-1636.

23. Kolavennu V, Zeng $\mathrm{L}$, Peng $\mathrm{H}$, et al. Targeting of RhoA/ROCK signaling ameliorates progression of diabetic nephropathy independent of glucose control. Diabetes 2008;57:714-723.

24. Yuen PS, Dunn SR, Miyaji T, et al. A simplified method for HPLC determination of creatinine in mouse serum. Am J Physiol Renal Physiol 2004;286:F1116-F1119.

25. Doi K, Okamoto K, Negishi K, et al. Attenuation of folic acid-induced renal inflammatory injury in platelet-activating factor receptordeficient mice. Am J Pathol 2006;168:1413-1424.

26. Morrissey JJ, Klahr S. Effect of AT2 receptor blockade on the pathogenesis of renal fibrosis. Am J Physiol 1999;276(Part 2):F39-F45.

27. Knosp WM, Saneyoshi C, Shou S, et al. Elucidation, quantitative refinement, and in vivo utilization of the HOXA13 DNA binding site. J Biol Chem 2007;282:6843-6853.

28. Park SH, Choi MJ, Song IK, et al. Erythropoietin decreases renal fibrosis in mice with ureteral obstruction: role of inhibiting TGF-beta-induced epithelial-to-mesenchymal transition. J Am Soc Nephrol 2007;18: 1497-1507.

29. Zhang A, Dong Z, Yang T. Prostaglandin D2 inhibits TGF-beta1induced epithelial-to-mesenchymal transition in MDCK cells. Am J Physiol Renal Physiol 2006;291:F1332-F1342.

30. Hartung R, Parapuram SK, Ganti R, et al. Vitreous induces heme oxygenase-1 expression mediated by transforming growth factor-beta and reactive oxygen species generation in human retinal pigment epithelial cells. Mol Vis 2007;13:66-78. 
31. Hay ED, Zuk A. Transformations between epithelium and mesenchyme: normal, pathological, and experimentally induced. Am J Kidney Dis 1995;26:678-690.

32. Reichert M, Muller T, Hunziker W. The PDZ domains of zonula occludens-1 induce an epithelial to mesenchymal transition of MadinDarby canine kidney I cells. Evidence for a role of beta-catenin/Tcf/Lef signaling. J Biol Chem 2000;275:9492-9500.

33. Bagnoli F, Buti L, Tompkins L, et al. Helicobacter pylori CagA induces a transition from polarized to invasive phenotypes in MDCK cells. Proc Natl Acad Sci USA 2005;102:16-16.

34. Collins R, Armitage J, Parish S, et al. MRC/BHF Heart Protection Study of cholesterol-lowering with simvastatin in 5963 people with diabetes: a randomised placebo-controlled trial. Lancet 2003;361:2005-2016.

35. Athyros VG, Mikhailidis DP, Papageorgiou AA, et al. The effect of statins versus untreated dyslipidaemia on renal function in patients with coronary heart disease. A subgroup analysis of the Greek atorvastatin and coronary heart disease evaluation (GREACE) study. J Clin Pathol 2004;57:728-734.

36. Koren MJ, Davidson MH, Wilson DJ, et al. Focused atorvastatin therapy in managed-care patients with coronary heart disease and CKD. Am J Kidney Dis 2009;53:741-750.

37. Tonelli M, Moye L, Sacks FM, et al. Effect of pravastatin on loss of renal function in people with moderate chronic renal insufficiency and cardiovascular disease. J Am Soc Nephrol 2003;14:1605-1613.

38. Shepherd J, Kastelein JJ, Bittner V, et al. Effect of intensive lipid lowering with atorvastatin on renal function in patients with coronary heart disease: the Treating to New Targets (TNT) study. Clin J Am Soc Nephrol 2007;2:1131-1139.

39. Colhoun HM, Betteridge DJ, Durrington PN, et al. Effects of atorvastatin on kidney outcomes and cardiovascular disease in patients with diabetes: an analysis from the Collaborative Atorvastatin Diabetes Study (CARDS). Am J Kidney Dis 2009;54:810-819.

40. Yokozawa $T$, Zheng PD, Oura $\mathrm{H}$, et al. Animal model of adenineinduced chronic renal failure in rats. Nephron 1986;44:230-234.

41. Cochrane AL, Kett MM, Samuel CS, et al. Renal structural and functional repair in a mouse model of reversal of ureteral obstruction. J Am Soc Nephrol 2005;16:3623-3630.

42. Yang J, Liu Y. Dissection of key events in tubular epithelial to myofibroblast transition and its implications in renal interstitial fibrosis. Am J Pathol 2001;159:1465-1475.
43. Zeisberg M, Hanai J, Sugimoto $H$, et al. BMP-7 counteracts TGF-beta1induced epithelial-to-mesenchymal transition and reverses chronic renal injury. Nat Med 2003;9:964-968.

44. $\mathrm{Xu} \mathrm{Y,} \mathrm{Wan} \mathrm{J,} \mathrm{Jiang} \mathrm{D,} \mathrm{et} \mathrm{al.} \mathrm{BMP-7} \mathrm{counteracts} \mathrm{TGF-beta1-induced}$ epithelial-to-mesenchymal transition in human renal proximal tubular epithelial cells. J Nephrol 2009;22:403-410.

45. Gould SE, Day M, Jones SS, et al. BMP-7 regulates chemokine, cytokine, and hemodynamic gene expression in proximal tubule cells. Kidney Int 2002;61:51-60.

46. Zeisberg $M$, Bottiglio $C$, Kumar N, et al. Bone morphogenic protein-7 inhibits progression of chronic renal fibrosis associated with two genetic mouse models. Am J Physiol Renal Physiol 2003;285: F1060-F1067.

47. Morrissey J, Hruska K, Guo G, et al. Bone morphogenetic protein-7 improves renal fibrosis and accelerates the return of renal function. J Am Soc Nephrol 2002;13(Suppl 1):S14-S21.

48. Zeisberg M. Bone morphogenic protein-7 and the kidney: current concepts and open questions. Nephrol Dial Transplant 2006;21: 568-573.

49. Tanaka M, Asada M, Higashi AY, et al. Loss of the BMP antagonist USAG-1 ameliorates disease in a mouse model of the progressive hereditary kidney disease Alport syndrome. J Clin Invest 2010;120: 768-777.

50. Neilson EG. Mechanisms of disease: fibroblasts-a new look at an old problem. Nat Clin Pract Nephrol 2006;2:101-108.

51. Zeisberg $M$, Shah AA, Kalluri R. Bone morphogenic protein-7 induces mesenchymal to epithelial transition in adult renal fibroblasts and facilitates regeneration of injured kidney. J Biol Chem 2005;280: 8094-8100.

52. Scott V, Morgan EA, Stadler HS. Genitourinary functions of Hoxa13 and Hoxd13. J Biochem 2005;137:671-676.

53. Mortlock DP, Innis JW. Mutation of HOXA13 in hand-foot-genital syndrome. Nat Genet 1997;15:179-180.

54. Fromental-Ramain C, Warot X, Messadecq N, et al. Hoxa-13 and Hoxd13 play a crucial role in the patterning of the limb autopod. Development 1996;122:2997-3011.

55. Williams $T M$, Williams $M E$, Heaton $\mathrm{JH}$, et al. Group 13 HOX proteins interact with the $\mathrm{MH} 2$ domain of R-Smads and modulate Smad transcriptional activation functions independent of HOX DNA-binding capability. Nucleic Acids Res 2005;33:4475-4484. 